\title{
A Systematic Review of the Effectiveness of Acceptance and Commitment Therapy (ACT) for Body Image Dissatisfaction and Weight Self-Stigma in Adults
}

\author{
Catrin Griffiths $^{1} \cdot$ Heidi Williamson ${ }^{1} \cdot$ Fabio Zucchelli $^{1}\left[\begin{array}{l}\text { [ } \\ \text { Nicole Paraskeva }\end{array}{ }^{1} \cdot\right.$ Tim Moss $^{1}$
}

Published online: 21 February 2018

(c) The Author(s) 2018. This article is an open access publication

\begin{abstract}
Body image dissatisfaction (BID) and weight self-stigma are prevalent and associated with physical and psychological illhealth. Acceptance and Commitment Therapy (ACT) is increasingly employed for both, yet little is known about its effectiveness. Searches of 12 databases identified six studies using online, face-to-face or self-help ACT interventions for BID or weight self-stigma, of varying duration and intensity. Their effectiveness and quality were evaluated. Two reported improved BID, three improved weight self-stigma, and one reported no impact on weight self-stigma. Methodological issues (small sample sizes, lack of allocation concealment, attention control and long-term follow up) impacted the validity of findings. Due to the small number of studies and poor study quality, the effectiveness of ACT for BID and weight self-stigma remains unclear. Nonetheless findings suggest psychological flexibility may facilitate reduction in BID and weight self-stigma and indicate that brief online as well as lengthy face-to-face delivery may be useful. Suggestions for further research are made.
\end{abstract}

Keywords Acceptance and Commitment Therapy $\cdot$ ACT $\cdot$ Body image $\cdot$ Weight self-stigma $\cdot$ Adults

\section{Introduction}

Around $61-93 \%$ of individuals report body image dissatisfaction (BID) (Diedrichs et al. in preparation; Liossi 2003). Growing recognition of the worldwide prevalence of BID and its negative impact on psychological and physical well-being has instigated demands for its recognition as a major public health concern (Bucchianeri and NewmarkSztainer 2014). Body image, defined as "one's perceptions

Catrin Griffiths

catrin.griffiths@uwe.ac.uk

Heidi Williamson

heidi3.Williamson@uwe.ac.uk

Fabio Zucchelli

fabio.Zucchelli@uwe.ac.uk

Nicole Paraskeva

Nicole.paraskeva@uwe.ac.uk

Tim Moss

tim.moss@uwe.ac.uk

1 Centre for Appearance Research, University of the West of England (UWE), Frenchay Campus, Coldharbour Lane, Bristol BS16 1QY, UK and attitudes in relation to one's own physical characteristics" (Cash and Fleming 2002, p. 455) such as weight, shape, height and skin colour, is a multi-dimensional construct that incorporates cognitive, affective, behavioural and perceptual facets. Negative body image, generally assessed using self-report measures of BID, is defined as "negative subjective evaluations of one's physical body" (Stice and Shaw 2002, p. 985) and can include negative appearancerelated thoughts (e.g., 'I am ugly'), feelings (e.g., selfconsciousness, appearance-anxiety) and behaviours (e.g. excessive appearance-checking). Those experiencing positive body image, often assessed using self-report measures of body appreciation, tend to respect and appreciate their body regardless of its appearance, engage in body-protective health behaviours and reject "unrealistic media appearance ideals" (Tylka and Homan 2015, p. 91).

BID can span a lifetime, and disproportionately affects women, although men are increasingly affected (Tiggemann 2004). BID is associated with heightened anxiety, depression, low self-esteem, reduced quality of life and risky health behaviours including disordered eating. Those affected may use crash-diets, laxative and diet pills and vomiting in attempt to change their body shape and weight (Bucchianeri and Newmark-Sztainer 2014; Ganem and Morera 2009). 
Identifying effective psychological interventions to reduce BID is therefore a priority for healthcare services.

Despite extreme and widespread social pressure to be thin in society, rates of obesity have substantially increased over the past 50 years (Finucane et al. 2011). Recent figures show $40.4 \%$ of women and $35 \%$ of men in the US are classed as obese and this is set to increase to $50 \%$ by 2030 (Wang et al. 2011). Consequently, the gap between the 'ideal' body size and the body of an overweight or obese person is growing. As the discrepancy between 'ideal' and actual body size increases, so does the risk of BID and weight-related selfstigma (Bessenoff and Snow 2006).

Overweight or obese people are commonly subjected to pervasive weight-related social discrimination and are often stereotyped as unattractive, lazy, immoral and dishonest (Latner et al. 2005). Individuals can internalise this stigma by accepting these beliefs, fearing stigma from others, engaging in self-devaluation, and holding weight-related self-stigmatising attitudes. This is collectively known as weight self-stigma (Lillis et al. 2010). Weight self-stigma is closely related to BID, and has been associated with a range of negative outcomes including feelings of isolation, poor psychological functioning, binge eating, depression and other psychiatric symptoms (Wott and Carels 2010). Rather than serving as a motivator for weight loss, those experiencing weight self-stigma are more likely to engage in unhealthy behaviours that impede weight loss. For example, Carels et al. (2009) identified that baseline levels of weight self-stigma predicted poorer self-monitoring, lower energy expenditure and greater calorie intake in a sample of overweight and obese adults participating in a weight loss programme. Thus it is vital for health interventions to target the psychological processes that influence weight gain, to help individuals become more accepting of their weight and eating-related experiences, and develop healthier behaviours and promote quality of life (Palmeira et al. 2017a).

Given the potential psychological difficulties associated with BID and weight self-stigma, and the role these can play in instigating and perpetuating disordered eating and obesity, it is vital to identify effective psychological interventions that ameliorate BID and weight self-stigma. Currently very little research has evaluated psychological interventions to reduce weight self-stigma. In contrast a range of psychosocial approaches have been evaluated that target BID. These are broadly grouped into interventions that use: cognitive behavioural therapy (CBT) to change negative or unpleasant thoughts, feelings and behaviours that influence negative body image; media literacy techniques to enable participants to critically evaluate and challenge media images and messages that promote female and male 'ideal body images' (i.e. the thin and muscular ideal); and psychoeducation, which aims to increase awareness of the factors that influence body image and knowledge of the impact negative body image has on psychological and physical well-being (Alleva et al. 2015). However, a meta-analytic review of 62 randomised controlled trials of body image interventions (which included CBT, media literacy and psychoeducation) found interventions engendered only small improvements in body image (Alleva et al. 2015). The authors acknowledged the need for large-scale, high-quality trials in this area and highlighted that relatively new interventions, such as mindfulness-based interventions, require empirical attention. Acceptance and Commitment Therapy (ACT; Hayes et al. 1999) is one such mindfulness-based intervention.

The rationale for using ACT in this context is based on empirical research that suggests psychological inflexibility (i.e. the tendency to rigidly attempt to control or avoid difficult internal experiences and to place extreme importance on the literal content of thoughts and feelings) has a significant relationship with both BID and weight self-stigma (Lillis et al. 2011). For example, Mancuso (2016) reported that body image inflexibility mediated the relationship between BID and both experiential avoidance and appearance-fixing in 156 women, meaning those with greater body image flexibility were less likely to employ these maladaptive coping strategies. Similarly Webb (2015) identified that older adolescent girls with greater body image psychological inflexibility were less likely to engage in body appreciation behaviours, and that this held when controlling for body mass index (BMI). In an obese sample, psychological flexibility and weight self-stigma was found to significantly predict health-related quality of life (Lillis et al. 2011). Lillis et al. also found that both psychological flexibility and weight self-stigma worked in combination (and independently) to fully mediate the relationship between BMI and healthrelated quality of life. These findings suggest that an increase in psychological flexibility relating to one's body image/ weight (i.e. being aware of, allowing and accepting difficult body image experiences) may reduce BID and weight self-stigma.

ACT aims to increase an individual's psychological flexibility in how they experience difficult thoughts and feelings about their physical appearance via six core processes of change collectively known as the "ACT Hexaflex": acceptance, cognitive defusion, contact with the present moment, self-as-context, value-driven behaviour, and commitment to value-driven behaviours (Hayes et al. 2006). ACT theory holds that the content of a "dysfunctional' thought (e.g. "I'm fat") is not inherently problematic and in need of modification. Rather, it is psychological inflexibility-identification with thoughts as self-evident facts (cognitive fusion) and avoidance of their associated aversive affective state (i.e. experiential avoidance) - that predicts negative affective and behavioural outcomes. ACT teaches mindfulness techniques and commitment to one's values to increase psychological 
flexibility. Webb et al. (2014) suggest that encouraging body image flexibility via mindful awareness and acceptance towards such thoughts, and using values to guide one's response (e.g. having negative body image thoughts but continuing to exercise at the gym by being guided by a value to stay healthy), will produce more adaptive weight loss behaviour and ultimately improve body image.

ACT can also enhance emotional well-being relevant to BID and weight self-stigma via the cultivation of a non-critical self-image as a result of non-judgemental self-awareness (Kristeller et al. 2006), which may be especially beneficial for those who negatively evaluate their bodies. ACT may also improve BID and weight self-stigma by encouraging exposure to difficult thoughts and emotions, leading to a desensitisation to difficult appearance-related thoughts and feelings and a subsequent reduction in distress. Lastly, developing acceptance may increase levels of meta-cognitive awareness of one's thought processes. This increases the likelihood of thoughts and feelings being simply observed, increasing a sense of self-control, which can reduce automatic reactions (Segal et al. 2002).

Currently little is known about the overall effectiveness of ACT for BID or weight self-stigma. However, ACT has been found to be effective at reducing selfstigma in other contexts where social discrimination is common, including among those with substance abuse (Luoma et al. 2012), sexual orientation difficulties (Yadavaia and Hayes 2012) and HIV (Skinta et al. 2014). Work exploring the impact of ACT in the highly related area of clinically diagnosed eating disorders is also still in its infancy. Manlick et al. (2012) literature review summarised empirical research illustrating that ACT is widely used and tested among those diagnosed with an eating disorder, and cited findings related to the impact of ACT on body image among this population. However, the authors did not evaluate the scientific quality of the findings. No systematic review to date has critically evaluated the effectiveness of research that has investigated ACT for BID or weight self-stigma. This is surprising given the widespread prevalence of both BID (61-93\%) and obesity (35-40.4\%) compared to rates of diagnosed eating disorders such as anorexia (5\%), bulimia (1-3\%) and binge eating disorder (1-3\%), and the increasing evidence of the predictive role BID and weight self-stigma play in these conditions. It also surprising given the growth of ACT within the field of clinically diagnosed eating disorders and body image. The aim of this systematic review was therefore to identify studies testing ACT interventions for BID and/or weight self-stigma in adults without a clinical eating disorder, evaluate the studies' quality, and synthesise the findings.

\section{Method}

This review used the PRISMA checklist for reporting systematic reviews (Moher et al. 2009) and guidance published by the Cochrane Collaboration (Higgins and Green 2011). No protocol for this systematic review is available publicly but further information can be provided by contacting the first author.

\section{Inclusion and Exclusion Criteria}

Studies were eligible if they included adult participants (over 18 years old) who had received an ACT intervention, whether individually or in a group, via single or multiple sessions, provided by a clinician or researcher in a hospital, community or university setting. Studies with and without a control were included to ensure the search was inclusive of all relevant literature. Studies had to include a quantitative outcome measure that assessed BID or weight self-stigma (either post-intervention or longer followup). Studies had to be published in English and could be published or unpublished. Studies targeting participants diagnosed with eating disorders, namely anorexia nervosa, bulimia nervosa or binge eating disorder, were excluded.

\section{Search Strategy}

A literature search on ACT for improving BID and/or weight self-stigma was conducted utilising the electronic databases Amed, Cinahl Plus, Medline, Psycarticles, PsycINFO, Web of Science, the Cochrane Library, Assia, British Humanities Index, IBSS, PILOTS and Social Services Abstracts. The search terms were ("acceptance" OR "defusion") AND ("body" OR "appearance" OR "weight" OR "shape" OR "eating" OR "visible difference*" OR "disfigure*" OR "overweight" OR "obesity" OR “obese"). The authors checked the search terms with two experts in the field of body image research to ensure for accuracy and comprehensiveness, resulting in the search term "disfigure*" being added. The terms were searched for within the title and abstract of articles. No starting time period was specified but records were searched up until October 2017. Forward and backward snowballing techniques were also applied (via Web of Science cited reference searches and reading reference lists from key papers), and a search of unpublished and grey papers was conducted to mitigate the effects of publication bias (Song et al. 2010). Unpublished or missing information was requested from the study's corresponding author when necessary. Two reviewers independently extracted data, and any discrepancies were discussed between reviewers and resolved through 


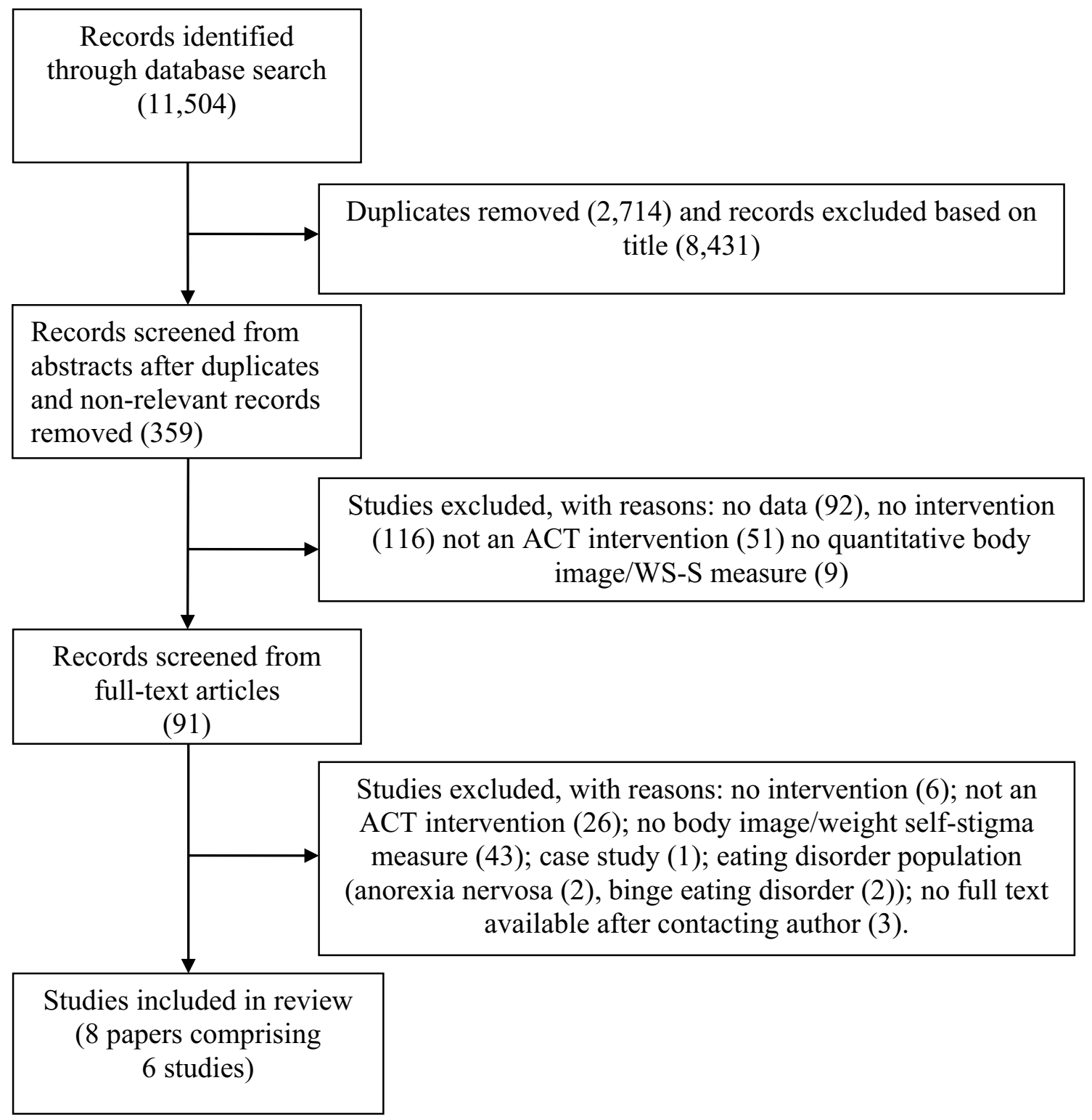

Fig. 1 Flow diagram of systematic selection of papers in review

consensus. Figure 1 shows the study search results and selection process.

\section{Assessment of Risk of Bias}

The methodological quality of each study was assessed by two reviewers according to the recommendations of the Cochrane Collaboration using the Cochrane risk of bias tool (Higgins and Green 2011). This tool assesses the generation of allocation sequence, allocation concealment, blinding of outcome assessors, completeness of follow-up data, selective reporting, and other sources of bias (see Fig. 2).

\section{Results}

\section{Study Characteristics}

The six individual studies in the review incorporated 351 individual participants (326 females and 25 males) and were published between 2009 and 2017 (Table 1). All were published in peer-reviewed journals apart from Fletcher's (2011) study, which was a doctorate thesis. The ethnicity of the participants was reported in three out of six studies (Fletcher 2011; Lillis et al. 2009; Levin et al. 2017) and the majority ethnic group was Caucasian. The average BMI of participants across the studies was 33.20 (range 22.38-38.01), which falls at the low end of the obese 


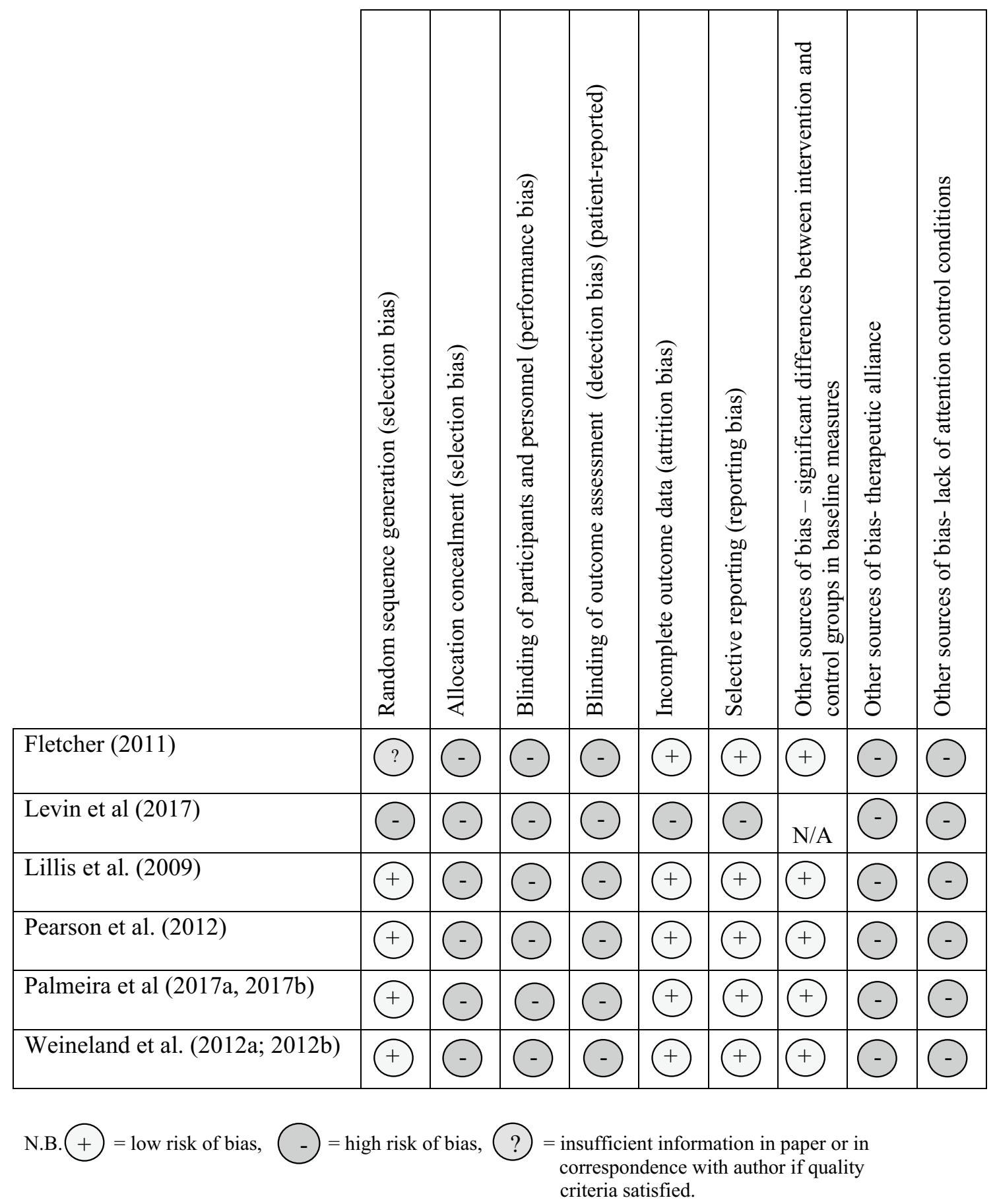

Fig. 2 The risk of bias of individual studies using the Cochrane risk of bias tool. N.B. low risk of bias, $=$ high risk of bias, $=$ insufficient information in paper or in correspondence with author if quality criteria satisfied

category. Four studies were conducted in the US (Fletcher 2011; Levin et al. 2017; Lillis et al. 2009; Pearson et al. 2012), Portugal (Palmeira et al. 2017a, b) and Sweden (Weineland et al. 2012a). Two studies included participants who had previously attended a weight-loss program (Fletcher 2011; Lillis et al. 2009); one recruited participants who self-reported weight self-stigma and who had previously participated in a weight loss program (Levin et al. 2017); one recruited participants actively enrolled in a weight loss program (Palmeira et al. 2017a, b); one recruited participants who had undergone bariatric surgery (Weineland et al. 2012a); and one enrolled women who self-reported BID (Pearson et al. 2012). 


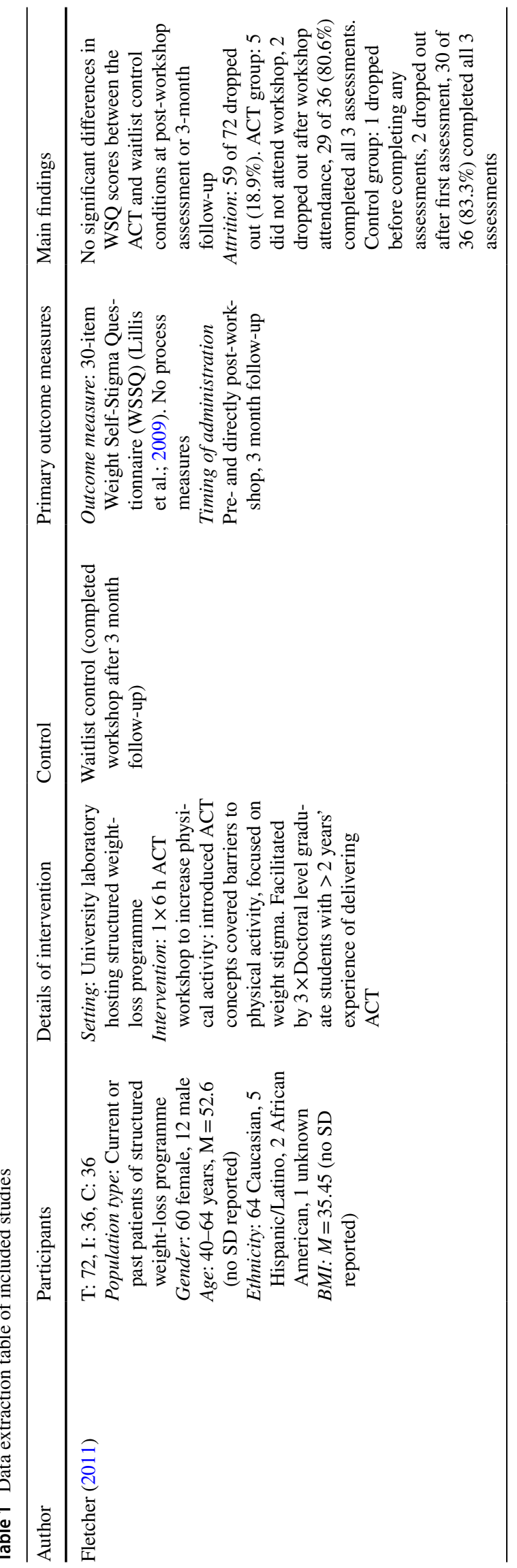




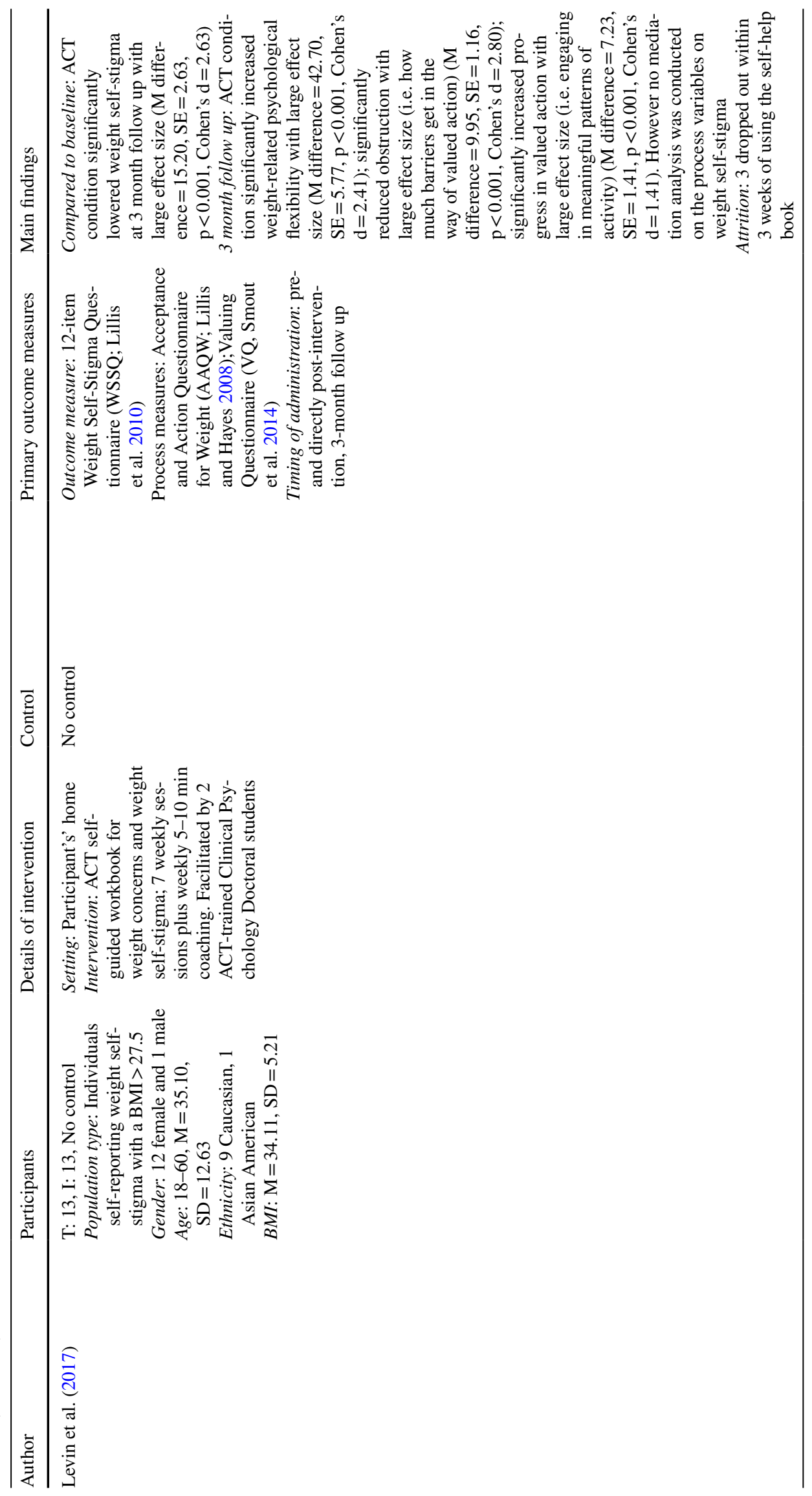




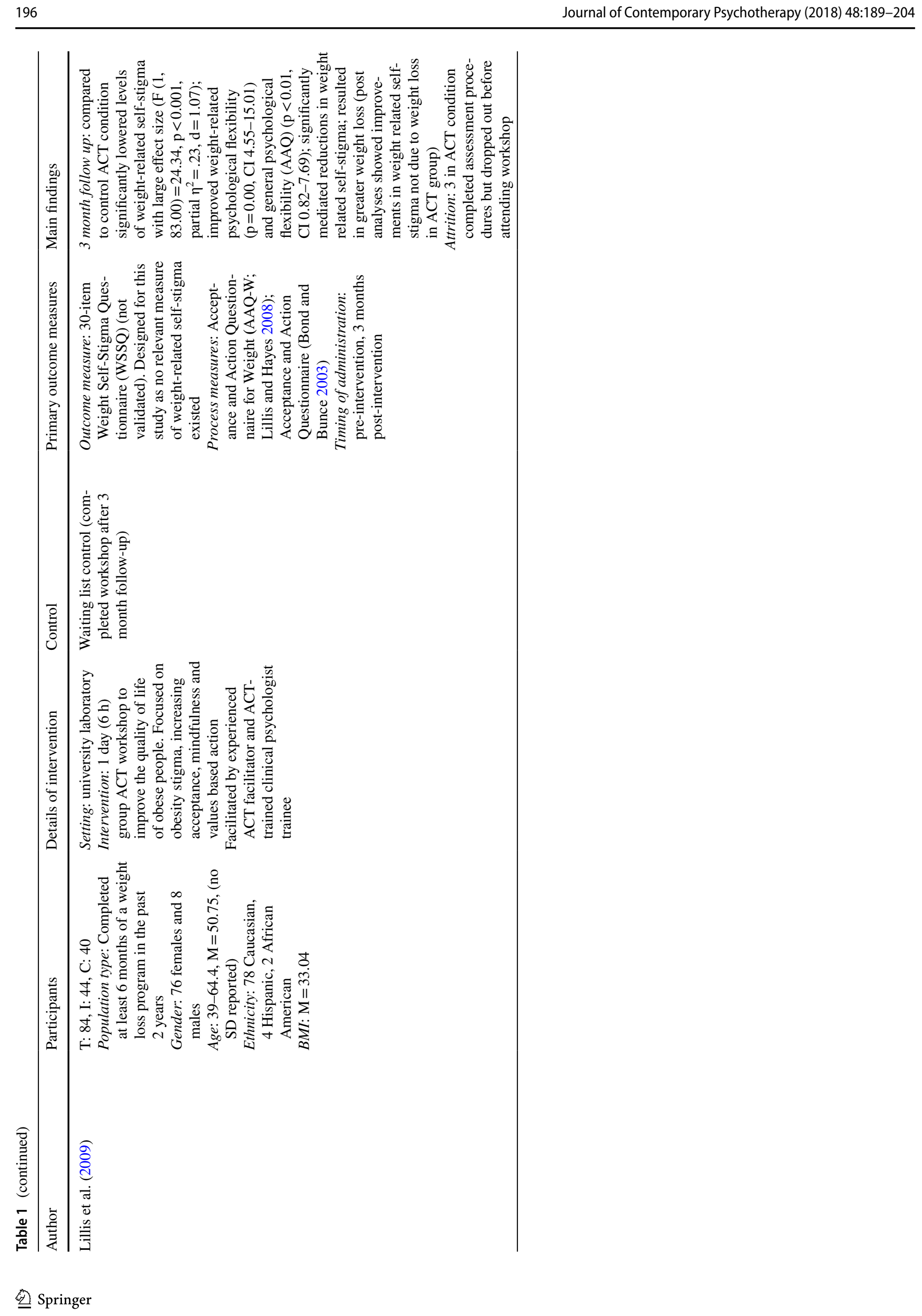




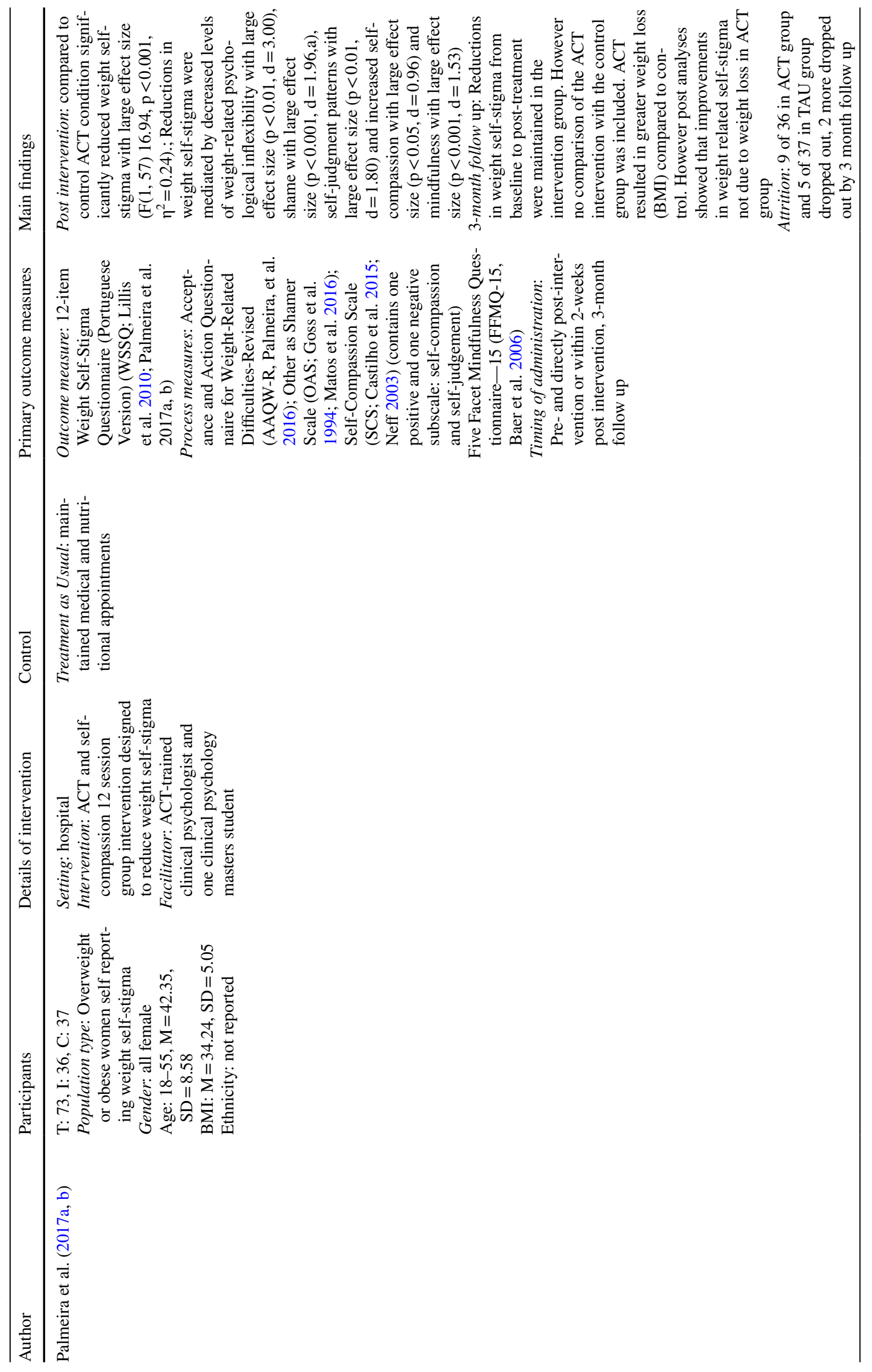




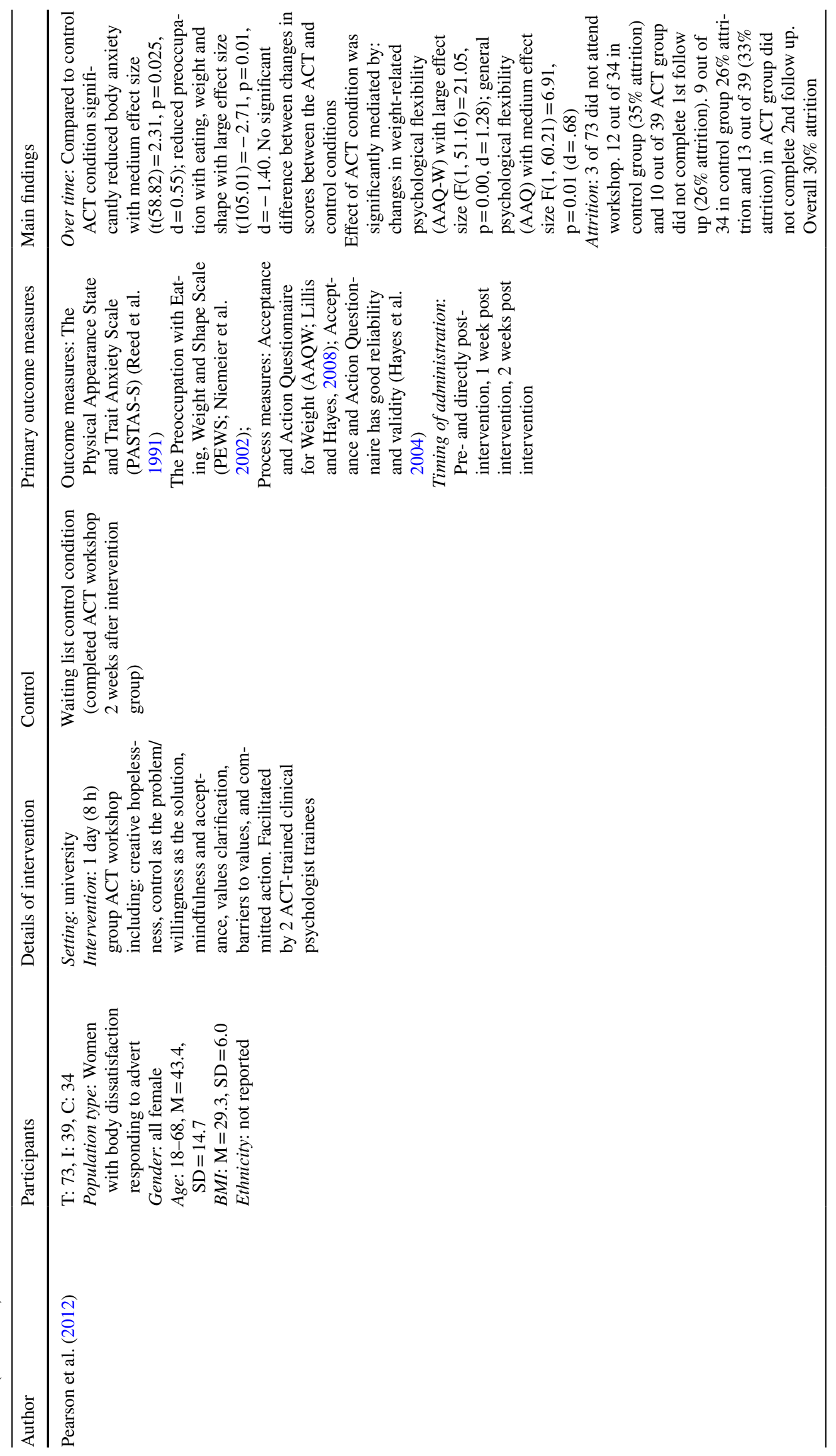




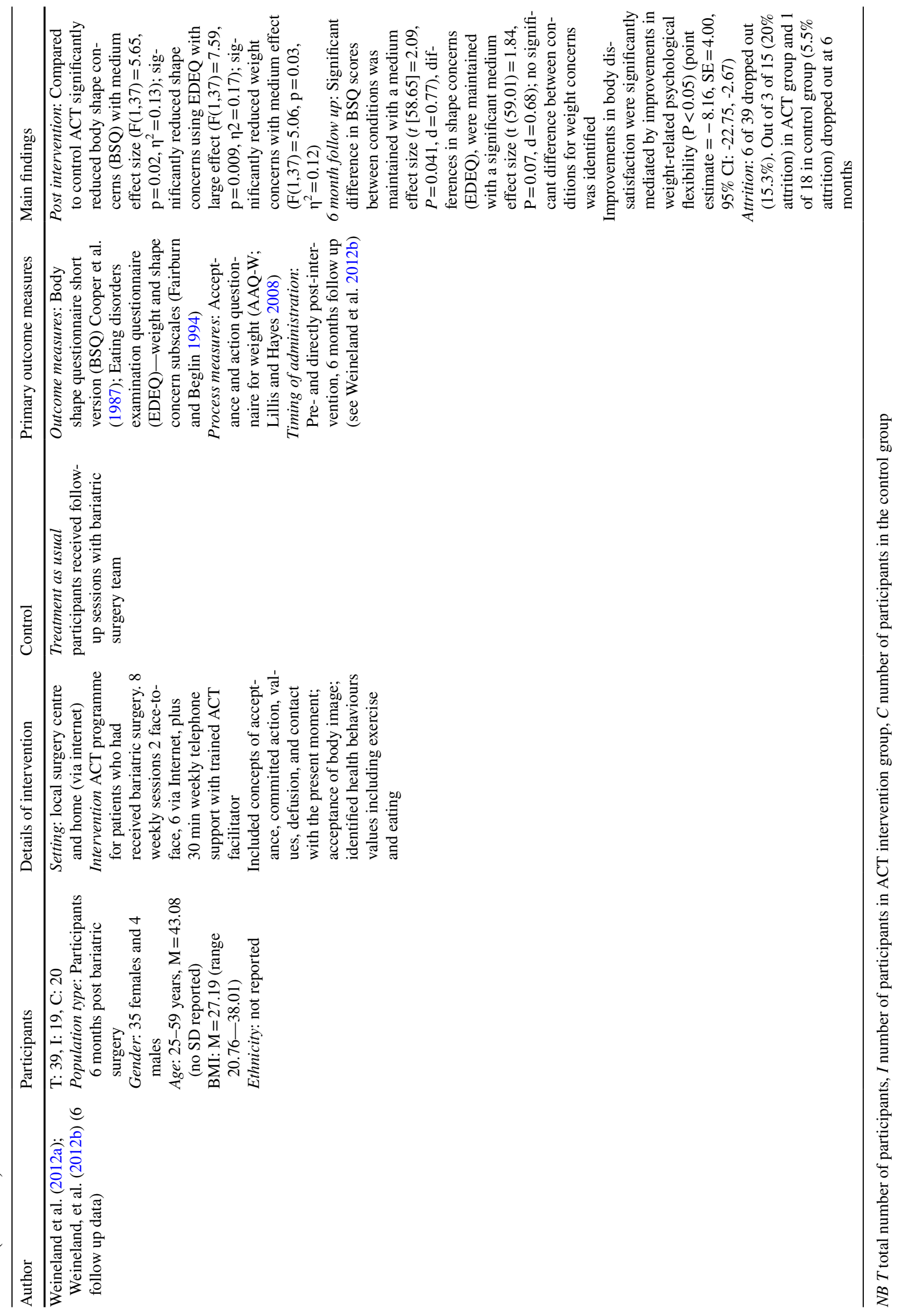


All six interventions used an ACT programme based on the original manual by Hayes et al. (1999) and were delivered by ACT-trained therapists (in conjunction with online delivery in Weineland et al. 2012a, b) or a self-help book called "The Diet Trap" (Lillis et al. 2014) in Levin et al's (2017) intervention. Two studies adopted a treatment as usual group (TAU) as a control (Palmeira et al. 2017a, b; Weineland et al. 2012a), and three studies used waiting list control groups. Levin et al's (2017) study had no control group.

Studies employed a variety of measures to assess BID or weight self-stigma. Pearson et al. (2012) used the Physical Appearance State and Trait Anxiety Inventory-State Version (PASTAS; Reed et al. 1991) and the Preoccupation with Eating, Weight, and Shape Scale (PEWS; Niemeier et al. 2002). Weineland et al. (2012a) used the weight and shape concerns subscales from the Eating Disorders Examination Questionnaire (EDEQ) and the Body Shape Questionnaire short version (BSQ; Cooper et al. 1987). Lillis et al. (2009) and Fletcher (2011) used the original 30-item Weight SelfStigma Questionnaire, which was designed by Lillis et al. specifically for their study. Levin et al. (2017) and Palmeira et al. (2017a, b) used the 12-item version of the Weight SelfStigma Questionnaire (Lillis et al. 2010). Post-intervention outcome measures were administered within different timeframes: Fletcher (2011) administered outcome measures at 1 week and 3 months post intervention; Lillis et al. (2009) repeated assessment at 3 month follow-up; Pearson et al. (2012) included 1- and 2-week follow-ups; Palmeira et al. (2017a, b) followed up within 2 weeks post-intervention and again at 3 months; Levin et al. (2017) followed up directly post-intervention and 3 month follow-up; and Weineland et al. (2012b) followed up directly post-intervention and at 6 months post-intervention.

All but two studies that utilised an RCT design described an appropriate method of randomisation: using computerbased randomisation (Palmeira et al. 2017a, b; Weineland et al. 2012a), coin flip (Pearson et al. 2012) and a random numbers table (Lillis et al. 2009). Levin et al. (2017) did not include a control group and therefore did not conduct randomisation of participants. Fletcher (2011) did not describe the method of randomisation, which resulted in an unclear risk of selection bias, increasing the likelihood of the exaggerated effect sizes. Methods of allocation concealment were not reported in any study and email communication with all authors identified that suitable methods were unlikely to have been used. Overall, lack of allocation concealment suggests high risk of selection bias and magnified reporting of effect sizes.

As is typical in psychological intervention research, blinding of participants was not possible in any study. Similarly, outcome assessors were not blinded to participants' condition in any study due to the self-reported nature of outcome measures. Palmeira et al. (2017a) blinded the clinical psychologists conducting data collection to the participants' assigned condition. However, participants were not blinded to the outcome assessment since they self-reported their answers to the questionnaires. Risk of performance and detection bias was therefore considered to be high. Attrition rates were 30\% directly post-intervention (Levin et al. 2017), $30 \%$ at 2 weeks (Pearson et al. 2012), 7.5, 15 and $18.9 \%$ at 3 months (Lillis et al. 2009, Palmeira et al. 2017a, b, and; Fletcher 2011, respectively) and $12 \%$ at 6 month follow-up (Weineland et al. 2012b). There were no significant differences in attrition rates between the intervention group and control in all studies that included a control, indicating the studies show an overall low risk of attrition bias.

All studies other than Levin et al., (2017) and Pearson et al. (2012) conducted power analyses. Of the five studies that included a control, Pearson et al. (2012), Palmeira et al. (2017a) and Weineland et al. (2012a) used an intention to treat approach to data analysis, and Lillis et al. (2009) and Fletcher (2011) used a per protocol approach. All studies reported the results of all measures described in their methods, and clearly described how the data was analysed and the number of participants included in the analysis, resulting in a low risk of selective reporting bias. Lillis et al. (2009) reported a significant difference at baseline between the ACT and control group in previous success at losing weight through dieting, but this was adequately addressed by the authors by including previous dieting success as a covariate in the analysis. Otherwise there were no significant differences between group outcome measure scores and other key variables at baseline. Sources of other bias were identified across studies. No studies included an attention control. Whether the benefits of the intervention were due to the ACT techniques specifically or as an effect of receiving an intervention of any kind is therefore unclear. The therapeutic alliance between the ACT facilitator/coach and participants may also have influenced intervention effects.

\section{Main Findings}

Despite adopting a broad inclusion criteria only a small number of eligible studies were identified for review. Duration of the ACT interventions varied (one-day workshops in three studies, a seven-session, eight-session and twelve-session programme in three studies), and a variety of measures targeting different constructs of body image and weight selfstigma were administered. This heterogeneity across studies rendered a meta-analysis unsuitable; a narrative synthesis was therefore applied.

The principle summary measures were the difference in means between the intervention and control groups' pre and post intervention scores. Effect sizes and p-values were reported. Of the six reviewed studies, analyses of changes in 
post-treatment outcome measures of BID and weight selfstigma revealed that four studies showed a significant effect of ACT compared to control, with reported Cohen's d effect sizes ranging from medium to large $(d=0.68-2.63$, where effect sizes of $d \geq 0.2$ are deemed small, $d \geq 0.5$ medium and $d \geq 0.8$ large, Cohen 1977) and partial eta squared effect sizes ranging from medium to large $\left(\eta^{2}=0.12-0.17\right)$ (Partial eta square effect sizes: $0.01=$ small, $0.06=$ medium, $0.14=$ large). Levin et al. (2017) did not include a control, however a large significant effect of ACT on weight selfstigma from pre to post intervention was identified. Fletcher (2011) found no significant differences between ACT and control. Taken together, the studies presented methodological issues including small sample sizes, lack of allocation concealment, reliance on self-report, and homogeneity of participants-mainly overweight and obese Caucasian women. These issues prevent conclusions being drawn to a wider population, including other ethnic groups, men and people with a healthy weight.

\section{The Effect of ACT on BID}

Two studies tested the effect of an ACT intervention on direct measures of BID. In the study conducted by Pearson et al. (2012), 73 female participants (aged 18-68, mean age $43.4, \mathrm{SD}=14.7$ ) who responded to an advert and self-reported with BID were randomly assigned to either a one-day ( 8 h) ACT workshop, or waiting list control plus self-monitoring. The ACT condition resulted in a significant medium reduction in body anxiety compared to control two weeks post-intervention. A significant large reduction in preoccupation with thoughts regarding eating, weight, and shape was also identified in the ACT condition over time. However there was no significant difference in preoccupation with thoughts regarding eating, weight, and shape between the ACT and waitlist control condition in scores over time.

Weineland et al. (2012a) included 39 participants (35 women and 4 men, aged 25-59) who had undergone bariatric surgery at least 6 months previously. Participants were randomly assigned to eight weekly sessions of ACT or to TAU control. Participants in the ACT condition were given two face-to-face sessions in a hospital surgery department and six internet sessions, with a 30 min weekly support session over the telephone. At post-intervention the ACT condition significantly reduced body shape concerns with a medium effect size compared to TAU control. The significant difference in body shape concerns between conditions was maintained at 6 month follow-up, with a medium effect size (Weineland et al. 2012b). The ACT condition had a significant medium effect on reducing post-intervention weight concerns compared to control. However, no significant difference between conditions for weight concerns was identified at 6 months post-intervention. Overall these two studies resulted in medium and large significant improvements BID measures.

\section{The Effect of ACT on Weight Self-Stigma}

Four studies (Lillis et al. 2009; Palmeira et al. 2017a; Levin et al. 2017; Fletcher 2011) investigated the impact of ACT on weight self-stigma in overweight and obese adults. Lillis et al. (2009) recruited 84 participants (76 female, mean age $=51.7)$ who had completed at least 6 months of any structured weight loss program in the previous 2 years. Participants were randomly assigned to a waiting list control group or an intervention group that received a one-day $(6 \mathrm{~h})$ ACT workshop using weight self-stigma as the focus. At 3 months post-intervention the ACT condition had a significant large effect on reducing weight self-stigma compared to control. The ACT group lost significantly more weight than the control. However, further analysis indicated that this weight loss was not responsible for the significant reductions in weight self-stigma.

Fletcher (2011) recruited 72 participants (60 female, mean age $=52.6$ ) who were current or previous patients of an established university laboratory-based structured weightloss program. Participants were randomly assigned to either a one day (6 h) ACT workshop designed to increase participants' physical activity, or to a waiting list control. The primary focus of the intervention was on physical activity rather than BID, however the workshop covered the role of weight self-stigma as a barrier to exercise. No significant differences were identified in weight self-stigma between conditions at post-intervention or at 3 month follow-up.

Palmeira et al's (2017a, b) study recruited 73 overweight or obese women aged 18-55 who reported weight selfstigma. Participants were randomly assigned to either a 12-session face-to-face ACT and self-compassion intervention designed to reduce weight self-stigma, or to a TAU control group. Compared to TAU, the ACT condition had a significant large effect on weight self-stigma post-intervention. In a follow-up study, reductions in weight self-stigma from baseline to post-treatment were maintained after 3 months (Palmeira et al. b). However, data reporting weight selfstigma levels in the control group at 3 month follow-up was not included. The ACT condition resulted in greater weight loss (BMI) compared to control. However, post-hoc analyses showed that improvements in weight related self-stigma were not due to weight loss in the ACT group.

Levin et al. (2017) recruited 13 participants ( 12 female) aged 18-60 struggling with weight self-stigma with a BMI of 27.5 or above. The intervention involved seven weekly ACT self-guided sessions using an ACT self-help book for weight self-stigma, plus weekly 5-10 min coaching sessions. At 3 month follow-up, the ACT condition showed a 
significant large effect on weight self-stigma compared to baseline.

All studies that utilised multiple weekly sessions found large significant effects in reducing weight self-stigma (Palmeira et al. 2017a, b; Levin et al. 2017). However, Levin et al's (2017) findings are limited by not including a control. Studies that used a single day intervention showed mixed results (Fletcher 2011; Lillis et al. 2009). Fletcher (2011) did not show a significant effect of ACT on weight self-stigma, whereas Lillis et al. (2009) found a large significant effect. It should be noted that Fletcher (2011) only dedicated a small part of the protocol to weight self-stigma, whereas Lillis et al's (2009) one-day ACT protocol specifically targeted weight self-stigma.

\section{Process Variables Results}

Five studies included ACT process outcome measures (i.e. psychometric measures which measure the key ACT processes which are targeted in ACT interventions: acceptance, cognitive defusion, contact with the present moment, self as context, value-driven behavior, and committed action towards value-driven behaviors) to test proposed mediating variables of the interventions' effects on body image or weight self-stigma (Levin et al. 2017; Lillis et al. 2009, Palmeira et al. b; Pearson et al. 2012; Weineland et al. 2012a) (see Table 1). Fletcher (2011) tested weight selfstigma as a process rather than an outcome variable, as the authors sought to test weight self-stigma as a mediator of increased physical activity (the target of the ACT intervention). Therefore no process variables were introduced to test the intervention's effect on weight-self stigma in this study. Of the two studies that examined BID, improvements in weight-specific psychological flexibility significantly mediated improvements in body image outcomes (Pearson et al. 2012; Weineland et al. 2012a). Pearson et al. (2012) found that improvements in general ACT processes also mediated improvements in body image.

Three out of the four studies that aimed to reduce weight self-stigma included ACT process measures. Lillis et al. (2009) found that increases in weight-related psychological flexibility and general psychological flexibility mediated the improvements in weight self-stigma in the ACT condition. Palmeira et al. (2017b) found that the significant reduction in weight self-stigma in the ACT conduction was mediated by decreased levels of weight-related psychological inflexibility, shame, self-judgment patterns and increased self-compassion and mindfulness. Levin et al. (2017) found significant reductions in weight-related psychological inflexibility, values obstruction (i.e. how much barriers get in the way of valued action) and increased progress towards valued action in the ACT condition. However, no mediation analysis was conducted between the process variables and weight self-stigma, leaving it unclear as to whether these ACT process variables mediated weight self-stigma (Levin et al. 2017).

\section{Discussion}

Overall the findings suggest that there is a lack of research investigating the effectiveness of ACT for improving body image and weight self-stigma. This review indicates that ACT for BID and weight self-stigma shows promise. However, due to the small number of studies and inconsistent findings, the effectiveness of ACT for BID and weight selfstigma remains unclear. The reviewed studies also presented methodological issues (small sample sizes, lack of allocation concealment, a variety of comparison groups, a lack of attention control and lack of long-term follow up) that impacted the validity of the findings.

\section{Intervention Duration and Format}

Overall the review identified that a variety of intervention durations (multiple weekly sessions vs. a one-day workshop) and formats (face-to-face, online and a self-help book) yielded similar results. All studies apart from Fletcher (2011) reported similar large effect sizes on BID or weight self-stigma, revealing no dose-response relationship. This suggests ACT therapists and researchers can be flexible in how they deliver ACT, and that self-help provided online or via literature can offer an effective resource for geographically remote patients.

\section{Process Variables}

Of the five studies that included process variables, all showed improvements in weight-related ACT processes in the intervention groups, which mediated improvements in BID and weight self-stigma in four studies. The two studies that included measures of general psychological flexibility reported that improvements in general ACT processes also mediated improvements in the body image measures. Another study found that other ACT-related process measures self-judgment patterns, increased self-compassion and mindfulness also mediated the ACT intervention effects. Future ACT studies may consider including both general and weight/appearance related ACT measures to allow for a more thorough investigation of ACT processes on intervention effects.

\section{Limitations and Future Directions}

Across the included studies, allocation concealment was not conducted during randomisation and no attention control was included. To reduce these potential biases, future studies should employ and report adequate allocation sequence 
concealment and include an attention control condition to enable a more thorough investigation of intervention effects.

A final limitation of the reviewed studies is the homogeneity of participants, who were adult and mainly overweight or obese white women. This prevents generalisations to a wider population. Recommendations for future work therefore include an investigation into the effectiveness of ACT for BID and weight self-stigma in men, children, teenagers and people from ethnic minority groups, those with a condition or injury that affects appearance, and those in 'normal' or 'underweight' BMI categories. Detailed investigation into the effectiveness of remotely-delivered ACT (e.g. via online methods) and a comparison of the effectiveness of ACT compared to other psychotherapies for BID and weight self-stigma would also be worthy of future investigation. In conclusion, this systematic review highlights that ACT interventions for BID and weight self-stigma shows promise, and investment in future research using larger-scale, higher quality randomized controlled trials is justified and required to confirm the effectiveness of ACT in this area.

Acknowledgements This research did not receive any specific grant from funding agencies in the public, commercial, or not-for-profit sectors.

\section{Compliance with Ethical Standards}

Conflict of interest All of the contributing authors declare that they have no conflict of interest.

Research Involving Human and Animal Participants This article does not contain any studies with human participants performed by any of the authors.

Informed Consent No informed consent was therefore required.

Open Access This article is distributed under the terms of the Creative Commons Attribution 4.0 International License (http://creativeco mmons.org/licenses/by/4.0/), which permits unrestricted use, distribution, and reproduction in any medium, provided you give appropriate credit to the original author(s) and the source, provide a link to the Creative Commons license, and indicate if changes were made.

\section{References}

Alleva, J. M., Sheeran, P., Webb, T. L., Martijn, C., \& Miles, E. (2015). A meta-analytic review of stand-alone interventions to improve body image. PLOS ONE, 10(9), e0139177.

Baer, R. A., Smith, G. T., Hopkins, J., Krietemeyer, J., \& Toney, L. (2006). Using self-report assessment methods to explore facets of mindfulness. Assessment, 13(1), 27-45.

Bessenoff, G. R., \& Snow, D. (2006). Absorbing society's influence: Body image self-discrepancy and internalized shame. Sex Roles, 54, 727-731.
Bond, F. W., \& Bunce, D. (2003). The role of acceptance and job control in mental health, job satisfaction, and work performance. Journal of Applied Psychology, 88(6), 1057.

Bucchianeri, M. M., \& Newmark-Sztainer, D. (2014). Body dissatisfaction: An overlooked public health concern. Journal of Public Mental Health, 13, 64-69.

Carels, R. A., Young, K. M., Wott, C. B., Harper, J., Gumble, A., Oehlof, M. W., \& Clayton, A. M. (2009). Weight bias and weight loss treatment outcomes in treatment-seeking adults. Annals of Behavioral Medicine, 37, 350-355.

Cash, T. F., \& Fleming, E. C. (2002). The impact of body image experiences: Development of the body image quality of life inventory. International Journal of Eating Disorders, 31(4), 455-460.

Castilho, P., Pinto-Gouveia, J., \& Duarte, J. (2015). Evaluating the multifactor structure of the long and short versions of the selfcompassion scale in a clinical sample. Journal of Clinical Psychology, 71(9), 856-870..

Cohen, J. (1977). Statistical power analysis for the behavioral sciences. New York: Academic Press.

Cooper, P. J., Taylor, M. J., Cooper, Z., \& Fairbum, C. G. (1987). The development and validation of the body shape questionnaire. International Journal of Eating Disorders, 6, 485-494.

Diedrichs, P. C., Halliwell, E., Craddock, N., Paraskeva, N., Bond, R., \& Rumsey, N. (in preparation). Body image in the UK: Examining the nature of body image concerns among a sample of 77000 British adults. Manuscript in preparation.

Fairburn, C. G., \& Beglin, S. J. (1994). Assessment of eating disorders: Interview or self-report questionnaire? International Journal of Eating Disorders, 16(4), 363-370.

Finucane, M. M., Stevens, G. A., Cowan, M. J., Danaei, G., Lin, J. K., Paciorek, C. J., Singh, G. M., Gutierrez, H. R., Lu, Y., Bahalim, A. N., \& Farzadfar, F. (2011). National, regional, and global trends in body-mass index since 1980: Systematic analysis of health examination surveys and epidemiological studies with 960 country-years and $9 \cdot 1$ million participants. The Lancet, 377, 557-567.

Fletcher, L. (2011). A Mindfulness and Acceptance-based Intervention for Increasing Physical Activity and Reducing Obesity (unpublished doctoral dissertation). University of Nevada, Reno.

Ganem, P. A., \& Morera, O. (2009). Does body dissatisfaction predict mental health outcomes in a sample of predominately Hispanic college students? Personality \& Individual Differences, 46, $557-561$.

Goss, K., Gilbert, P., \& Allan, S. (1994). An exploration of shame measures-I: The other as Shamer scale. Personality and Individual differences, 17(5), 713-717.

Hayes, S. C., Luoma, J. B., Bond, F. W., Masuda, A., \& Lillis, J. (2006). Acceptance and commitment therapy: Model, processes and outcomes. Behaviour Research and Therapy, 44(1), 1-25.

Hayes, S. C., Strosahl, K. D., \& Wilson, K. G. (1999). Acceptance and commitment therapy: An experiential approach to behavior change. New York: Guilford Press.

Hayes, S. C., Strosahl, K., Wilson, K. G., Bissett, R. T., Pistorello, J., Toarmino, D., Polusny, M. A., Dykstra, T. A., Batten, S. V., Bergan, J., \& Stewart, S. H. (2004). Measuring experiential avoidance: A preliminary test of a working model. The Psychological Record, 54(4), 553-578.

Higgins, J. P. T., \& Green, S. (2011). Cochrane Handbook for systematic reviews of interventions version 5.1.0 [updated March 2011].

Kristeller, J., Baer, R., \& Quillian-Wolever, R. (2006). Mindfulnessbased approaches to eating disorders. In R. A. Baer (Ed.), Mindfulness-based treatment approaches. (pp. 75-91). San Diego: Elsevier.

Latner, J. D., Stunkard, A. J., \& Wilson, G. T. (2005). Stigmatized students: Age, sex, and ethnicity effects in the stigmatization of obesity. Obesity Research, 13, 1226-1231. 
Levin, M. E., Potts, S., Haeger, J., \& Lillis, J. (2017). Delivering acceptance and commitment therapy for weight self-stigma through guided self-help: Results from an open pilot trial. Cognitive and Behavioral Practice (Online first).

Lillis, J., Dahl, J., \& Weineland, S. M. (2014). The diet trap: Feed your psychological needs and end the weight loss struggle using acceptance and commitment therapy. Oakland: New Harbinger Publications.

Lillis, J., \& Hayes, S. C. (2008). Measuring avoidance and inflexibility in weight related problems. International Journal of Behavioral Consultation and Therapy, 4(4), 348.

Lillis, J., Hayes, S. C., Bunting, K., \& Masuda, A. (2009). Teaching acceptance and mindfulness to improve the lives of the obese: A preliminary test of a theoretical model. Annals of Behavioral Medicine, 37, 58-69.

Lillis, J., Levin, M. E., \& Hayes, S. (2011). Exploring the relationship between BMI and health-related quality of life: A pilot study of the impact of weight self-stigma and experiential avoidance. Journal of Health Psychology, 16, 722-727.

Lillis, J., Luoma, J. B., Levin, M. E., \& Hayes, S. C. (2010). Measuring weight self-stigma: The weight self-stigma questionnaire. Obesity, 18, 971-976.

Liossi, C. (2003) Appearance related concerns across the general and clinical populations. Unpublished thesis, City University, London.

Luoma, J. B., Kohlenberg, B. S., Hayes, S. C., \& Fletcher, L. (2012). Slow and steady wins the race: A randomized clinical trial of acceptance and commitment therapy targeting shame in substance use disorders. Journal of Consulting and Clinical Psychology, $80,43-53$.

Mancuso, S. G. (2016). Body image inflexibility mediates the relationship between body image evaluation and maladaptive body image coping strategies. Body Image, 16, 28-31.

Manlick, C. F., Cochran, S. V., \& Koon, J. (2012). Acceptance and commitment therapy for eating disorders: Rationale and literature review. Journal of Contemporary Psychotherapy, 8, 1-8.

Matos, M., Pinto-Gouveia, J., \& Duarte, C. (2016) Other as Shamer. Versão portuguesa e propriedades psicométricas de uma medida de vergonha externa [Other as Shamer: Portuguese version and psychometric properties of a measure of external shame]. Manuscript submitted for publication.

Moher, D., Liberati, A., Tetzlaff, J., \& Altman, D. G. (2009). Preferred reporting items for systematic reviews and meta-analyses: The PRISMA statement. Annals of Internal Medicine, 151, 264-269.

Neff, K. D. (2003). The development and validation of a scale to measure self-compassion. Self and Identity, 2(3), 223-250.

Niemeier, H. M., Craighead, L. W., Pung, M. A., \& Elder, K. A. (2002). Reliability, validity and sensitivity to change of the Preoccupation with Eating Weight, and Shape Scale. Annual meeting of the Association of the Advancement of Behavior Therapy, Reno, NV.

Palmeira, L., Cunha, M., Pinto-Gouveia, J., Carvalho, S., \& Lillis, J. (2016). New developments in the assessment of weight-related experiential avoidance (AAQW-Revised). Journal of Contextual Behavioral Science, 5(3), 193-200.

Palmeira, L., Pinto-Gouveia, J., \& Cunha, M. (2017a). Exploring the efficacy of an acceptance, mindfulness \& compassionate-based group intervention for women struggling with their weight $(\mathrm{Kg}-$ Free): A randomized controlled trial. Appetite, 112, 107-116.

Palmeira, L., Cunha, M., \& Pinto-Gouveia, J. (2017b). Processes of change in quality of life, weight self-stigma, body mass index and emotional eating after an acceptance-, mindfulness-and compassion-based group intervention (Kg-Free) for women with overweight and obesity. Journal of Health Psychology, 2017, 1359105316686668.

Pearson, A. N., Follette, V. M., \& Hayes, S. C. (2012). A pilot study of acceptance and commitment therapy as a workshop intervention for body dissatisfaction and disordered eating attitudes. Cognitive and Behavioral Practice, 19, 181-197.

Reed, D. L., Thompson, J. K., Brannick, M. T., \& Sacco, W. P. (1991). Development and validation of the physical appearance state and trait anxiety scale (PASTAS). Journal of Anxiety Disorders, 5, 323-332.

Segal, Z. V., Williams, J. M. G., \& Teasdale, J. D. (2002). Mindfulnessbased cognitive therapy for depression: A new approach to preventing relapse. New York: Guilford Press.

Skinta, M. D., Brandrett, B. D., Schenk, W. C., Wells, G., \& Dilley, J. W. (2014). Shame, self-acceptance and disclosure in the lives of gay men living with HIV: An interpretative phenomenological analysis approach. Psychology \& Health, 29, 583-597.

Smout, M., Davies, M., Burns, N., \& Christie, A. (2014). Development of the valuing questionnaire (VQ). Journal of Contextual Behavioral Science, 3(3), 164-172.

Song, F., Parekh, S., Hooper, L., Loke, Y. K., Ryder, J., Sutton, A. J., Hing, C., Kwok, C. S., Pang, C., \& Harvey, I. (2010). Dissemination and publication of research findings: An updated review of related biases. Health Technology Assessment, 14, 1-193.

Stice, E., \& Shaw, H. E. (2002). Role of body dissatisfaction in the onset and maintenance of eating pathology. A synthesis of research findings. Journal of Psychosomatic Research, 53, 985-993.

Tiggemann, M. (2004). Body image across the adult life span: Stability and change. Body Image, 1, 29-41.

Tylka, T. L., \& Homan, K. J. (2015). Exercise motives and positive body image in physically active college women and men: Exploring an expanded acceptance model of intuitive eating. Body Image, 15, 90-97.

Wang, Y. C., McPherson, K., Marsh, T., Gortmaker, S. L., \& Brown, M. (2011). Health and economic burden of the projected obesity trends in the USA and the UK. The Lancet, 378, 815-825.

Webb, J. B. (2015). Body image flexibility contributes to explaining the link between body dissatisfaction and body appreciation in White college-bound females. Journal of Contextual Behavioral Science, 4, 176-183.

Webb, J. B., Butler-Ajibade, P., \& Robinson, S. A. (2014). Considering an affect regulation framework for examining the association between body dissatisfaction and positive body image in Black older adolescent females: Does body mass index matter? Body Image, 11, 426-437.

Weineland, S., Arvidssonb, D., Thanos, P., Kakoulidisb, T. P., \& Dahla, J. (2012a) Acceptance and commitment therapy for bariatric surgery patients, a pilot RCT. Obesity Research \& Clinical Practice, 6, e21-e30.

Weineland, S., Hayes, S. C., \& Dahl, J. (2012b). Psychological flexibility and the gains of acceptance-based treatment for post-bariatric surgery: Six-month follow-up and a test of the underlying model. Clinical Obesity, 2, 15-24.

Wott, C. B., \& Carels, R. A. (2010). Overt weight stigma, psychological distress and weight loss treatment outcomes. Journal of Health Psychology, 15, 608-614.

Yadavaia, J. E., \& Hayes, S. C. (2012). Acceptance and commitment therapy for self-stigma around sexual orientation: A multiple baseline evaluation. Cognitive and Behavioral Practice, 19, 545-559. 